Boundless Corporation Title: SBIR Phase I Final Report, Nanowire Cathode Material for Li-Ion Batteries Revision Date: August 31, 2004

\title{
NANOWIRE CATHODE MATERIAL FOR LITHIUM-ION BATTERIES
}

\author{
SBIR Phase I Final Report \\ Period of Performance: July 21, 2003 - April 20, 2004
}

Date of Issuance: July 2004

Author: John B. Olson

Boundless Corporation

5445 Conestoga Court, Suite 1B

Boulder, CO 80301

$\underline{\text { www.boundlesscorp.com }}$

\section{SBIR/STTR RIGHTS NOTICE}

These SBIR/STTR data are furnished with SBIR/STTR rights under Grant No. DEFG02-03ER83614. For a period of 4 years after acceptance of all items to be delivered under this grant, the Government agrees to use these data for Government purposes only, and they shall not be disclosed outside the Government (including disclosure for procurement purposes) during such period without permission of the grantee, except that, subject to the foregoing use and disclosure prohibitions, such data may be disclosed for use by support contractors. After the aforesaid 4-year period the Government has a royalty-free license to use, and to authorize others to use on its behalf, these data for Government purposes, but is relieved of all disclosure prohibitions and assumes no liability for unauthorized use of these data by third parties. This Notice shall be affixed to any reproductions of these data in whole or in part. 


\section{Executive Summary}

This project involved the synthesis of nanowire $\gamma-\mathrm{MnO}_{2}$ and characterization as cathode material for high-power lithium-ion batteries for EV and HEV applications. The nanowire synthesis involved the edge site decoration nanowire synthesis developed by Dr. Reginald Penner at UC Irvine (a key collaborator in this project). Figure 1 is an SEM image showing $\gamma-\mathrm{MnO}_{2}$ nanowires electrodeposited on highly oriented pyrolytic graphite (HOPG) electrodes.

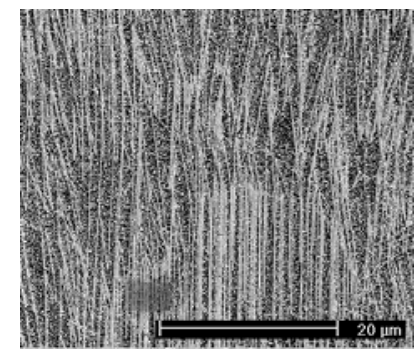

Figure 1. $\gamma-\mathrm{MnO}_{2}$ nanowires grown on HOPG electrodes (SEM image). Nanowires have diameters of approximately $100 \mathrm{~nm}$ and grow at edge sites of the graphite.

This technique is unique to other nanowire template synthesis techniques in that it produces long ( $>500 \mathrm{um}$ ) nanowires which could reduce or eliminate the need for conductive additives due to intertwining of fibers. Nanowire cathode for lithium-ion batteries with surface areas 100 times greater than conventional materials can enable higher power batteries for electric vehicles (EVs) and hybrid electric vehicles (HEVs).

The synthesis of the $\gamma-\mathrm{MnO}_{2}$ nanowires was successfully achieved. However, it was not found possible to co-intercalate lithium directly in the nanowire synthesis. Based on input from proposal reviewers, the scope of the project was altered to attempt the conversion into spinel $\mathrm{LiMn}_{2} \mathrm{O}_{4}$ nanowire cathode material by solid state reaction of the $\gamma-\mathrm{MnO}_{2}$ nanowires with $\mathrm{LiNO}_{3}$ at elevated temperatures. Attempts to perform the conversion on the graphite template were unsuccessful due to degradation of the graphite apparently caused by oxidative attack by $\mathrm{LiNO}_{3}$.

Emphasis then shifted to quantitative removal of the nanowires from the graphite, followed by the solid state reaction. Attempts to quantitatively remove the nanowires by several techniques were unsatisfactory due to co-removal of excess graphite or poor harvesting of nanowires.

Intercalation of lithium into $\gamma-\mathrm{MnO}_{2}$ electrodeposited onto graphite was demonstrated, showing a partial demonstration of the $\gamma-\mathrm{MnO}_{2}$ material as a lithium-ion battery cathode material.

Assuming the issues of nanowires removal can be solved, the technique does offer potential for creating high-power lithium-ion battery cathode needed for advanced EV and HEVs. Several technical advancements will still be required to meet this goal, and are likely topics for future SBIR feasibility studies. 


\section{Project Objectives}

The end objective of Phase I was to demonstrate the synthesis, characterization and performance feasibility of a new high power lithium battery nanowire cathode material. Three interim objectives that supported the above end objective are summarized below.

1. Evaluate synthesis conditions to optimize properties of nanowire materials. Characterize crystal and physical properties. We sought to answer the question of whether or not we could synthesize $\gamma-\mathrm{MnO}_{2}$ nanowires with properties suitable for cathode use.

The synthesis methods involved a novel nanowire technology that needed to be proven and refined for cathode candidate materials. The initial effort was on $\gamma-\mathrm{MnO}_{2}$. Since this material was capable of nanowire synthesis, focus remained on it. A contingency plan for the synthesis of $\mathrm{MoO}_{2}$ was also proposed, since the Penner group had extensive prior experience with $\mathrm{MoO}_{2}$.

2. Formulate cathode materials and intercalate lithium (if not formed in-situ). We sought to answer the question of whether or not we could intercalate lithium into the cathode in a single-step process as part of the nanowire synthesis, and to determine if the nanowires could increase cathode conductivity sufficiently to eliminate the need for carbon black.

The formulation work would consist in determining the appropriate matrix of PVDF and carbon black. It is likely that the carbon black may be reduced due to the excellent interconnectivity of the nanowires. If we were not able to successfully insert lithium as part of the electrodeposition of the nanowires, it would be necessary to insert the lithium electrochemically in a subsequent step during formulation.

3. Evaluate their use as cathodes in lithium-ion batteries. We had sought to answer the question of whether or not the new cathodes would support battery power and capacity levels that were as high or higher than conventional cathodes.

The results of objectives $1 \& 2$ were proposed be used to evaluate the potential capacity and power of the technology using lithium-ion pouch type batteries. Lithium metal reference electrodes may have been used to isolate the behavior of the nanowire active material cathodes. 


\section{Synthesis of $\mathrm{Y}-\mathrm{MnO}_{2}$ Nanowires Successful}

The first interim objective of the Phase I project was successfully accomplished: $\gamma-\mathrm{MnO}_{2}$ nanowires were synthesized and characterized. Figure 2 shows scanning electron microscope (SEM) images of three different nanowire depositions.

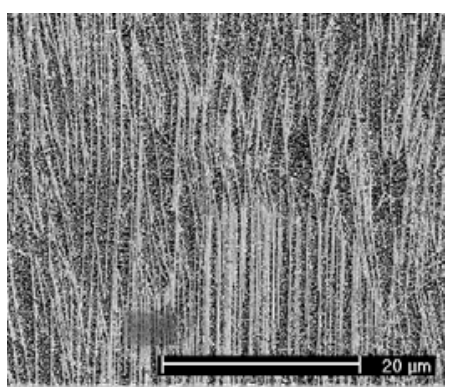

A.

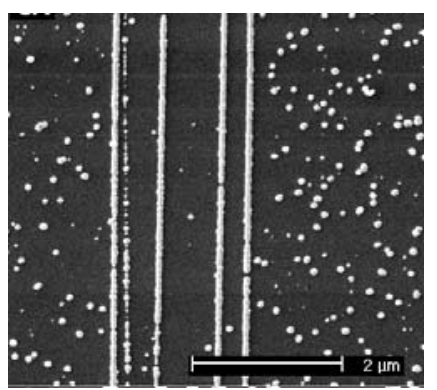

B.

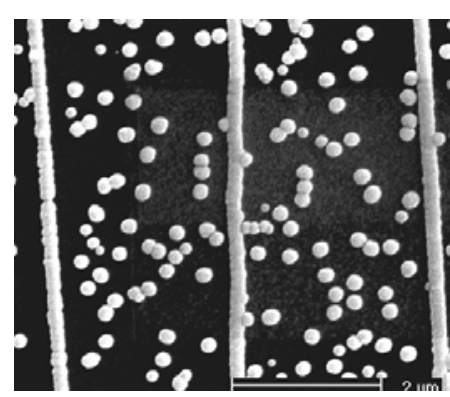

C.

Figure 2. SEM images of nanowire $\gamma-\mathrm{MnO}_{2}$ reductively electrodeposited onto HOPG electrodes: A) Nanowires deposited potentiostatically. B) Higher magnification of nanowires deposited at higher voltage for shorter time period. C) Nanowires deposited galvanostatically.

In addition to nanowires, some nanoparticles were also formed. Nanowires were successfully formed with either potentiostatic or galvanostatic control.

The nanowires were characterized by energy dispersive x-ray and a typical result is shown in Figure 3.

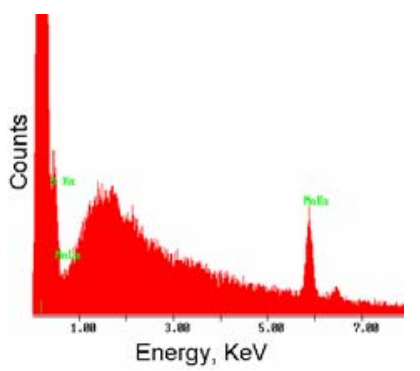

Figure 3. Energy dispersive x-ray spectrum showing strong peaks at $5.9 \mathrm{KeV}$ for manganese and $0.53 \mathrm{KeV}$ for oxygen, indicative of

$\mathrm{MnO}_{2}$.

The identity of the nanowires was further established using selected area electron diffraction on a film sample deposited under the same conditions as the nanowires and dehydrated at $300^{\circ} \mathrm{C}$ under nitrogen. The diffraction pattern and lattice spacing are shown in Figure 4. 


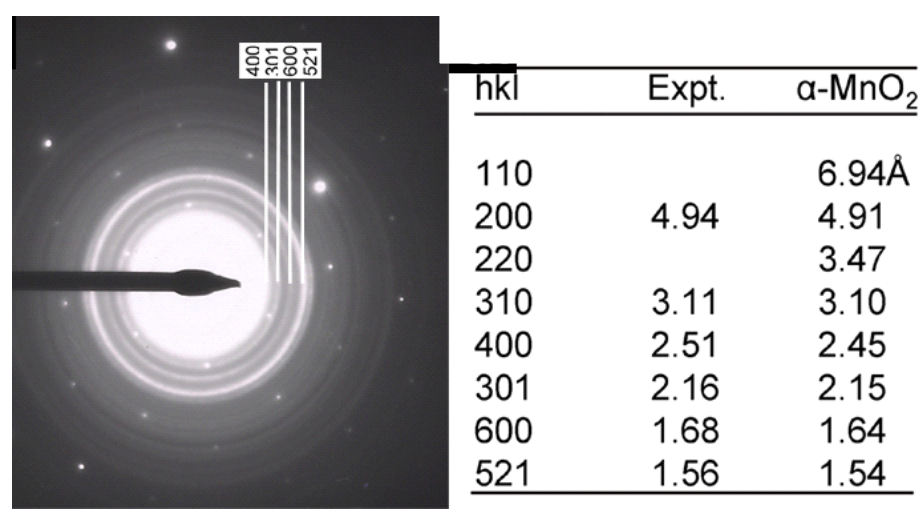

Figure 4. Selected area electron diffraction pattern and results for a film deposited under identical conditions to the nanowires. $\gamma-\mathrm{MnO}_{2}$ is conclusively identified.

The technology to grow the nanowires was then transferred from UCI to the Boundless labs. This was successfully accomplished after several iterative efforts, and Boundless gained the capability to scale the lab fabrication of nanowires up considerably by using galvanostatic control techniques and a Maccor multichannel battery cycler.

\section{Direct Electrodeposition of $\mathrm{LiMnO}_{2}$}

Effort then switched to attempts to electrodeposit $\mathrm{LiMnO}_{2}$ directly, however these attempts were unsuccessful. The $\gamma-\mathrm{MnO}_{2}$ deposits obtained from $\mathrm{LiNO}_{3}$-containing electrolyte did not incorporate lithium in any detectable amount as determined by electron diffraction.

\section{Attempts to Form Spinel LiMn $\underline{2}_{2} \underline{O}_{4}$}

We first attempted to get the spinel $\mathrm{LiMn}_{2} \mathrm{O}_{4}$ with solid state reactions with $\mathrm{LiNO}_{3}$ at temperatures of $500^{\circ} \mathrm{C}$, with the nanowires still attached to the HOPG. This procedure was based on literature accounts ${ }^{(1)}$.

Unfortunately, this procedure was unsuccessful; the graphite surface was etched and the nanowires had disappeared. An SEM image of the etched graphite surface is shown in Figure 5. 


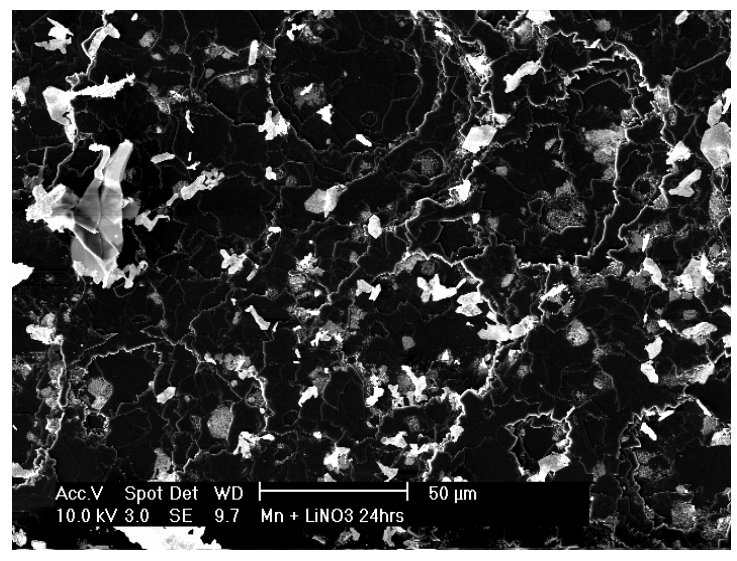

Figure 5. The etched surface of the HOPG following initial attempts to convert $\gamma-\mathrm{MnO}_{2}$ to spinel $\mathrm{LiMn}_{2} \mathrm{O}_{4}$ by solid state reaction with $\mathrm{LiNO}_{3}$ at $500^{\circ} \mathbf{C}$.

It was initially believed the HOPG had been attacked by oxygen, but later determined that a reaction with the $\mathrm{LiNO}_{3}$ was responsible.

Another attempt was made at $260^{\circ} \mathrm{C}$, which is just above the melting point of $\mathrm{LiNO}_{3}$, for 36 hours. Although the nanowires remained intact and the HOPG was not attacked, electron diffraction gave the same pattern as the unreacted nanowires, indicating the spinel form had not been created.

Next a series of experiments were performed to determine the maximum temperature at which the HOPG is stable. This was found to be just under $300^{\circ} \mathrm{C}$. Above $300^{\circ} \mathrm{C}$ the $\mathrm{HOPG}$ was attacked, but only in the presence of $\mathrm{LiNO}_{3}$. No spinel was formed at this temperature limit either. The lowest temperature reported in the literature for formation of the spinel was $370^{\circ} \mathrm{C}$.

At this point, we shifted our attention to the removal of the nanowires from the HOPG surface so we could react the nanowires into the spinel form without interference from the HOPG substrate. These efforts will be described later.

\section{Intercalation of Lithium into Dehydrated $\mathrm{y}-\mathrm{MnO}_{2}$}

A major objective of Task 4 as stated in our Phase I proposal and part of the second sub-objective was to demonstrate that we could electrochemically intercalate lithium into dehydrated $\gamma-\mathrm{MnO}_{2}$ nanowires. We successfully accomplished this objective in Phase I, though not in the manner originally proposed (removal of the lithiated nanowires and crystal characterization).

Instead we used electrochemical data as evidence for reversible lithium intercalation into $\gamma-\mathrm{MnO}_{2}$ films and nanowires. Initially, a $\gamma-\mathrm{MnO}_{2}$ film was deposited on a platinum electrode with the same conditions used for growing nanowires. This film (still on the electrode) was dehydrated and placed in an electrochemical cell with lithium-ion battery electrolyte. $\mathrm{A} \mathrm{Ag} / \mathrm{AgCl}$ electrode, which is about $2.9 \mathrm{~V}$ positive relative to lithium, was used for a reference electrode. A cyclic voltammetry scan from $0.8 \mathrm{~V}$ to $-1 \mathrm{~V}$ at $10 \mathrm{mV} / \mathrm{s}$ was done. The results are shown in Figure 6. 


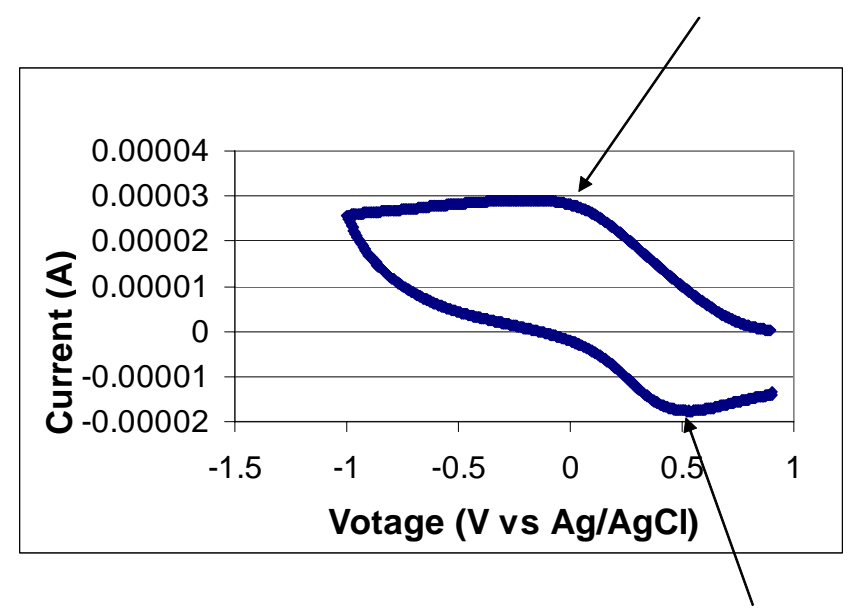

\section{Lithium de-intercalation}

Figure 6. Cyclic voltammetry results showing intercalation into a $\gamma-\mathrm{MnO}_{2}$ film deposited under the same conditions as the nanowires. Position of current peaks in the scan match literature values.

The cyclic voltammetry (CV) shows good evidence for the intercalation and de-intercalation of lithium in the $\gamma-\mathrm{MnO}_{2}$ film. The peaks on the $\mathrm{CV}$ correspond closely to those indicated in literature references ${ }^{(32)}$. A scan on a clean platinum electrode showed no peaks.

Next, the same analysis was performed on dehydrated nanowires still on the HOPG electrode. CV results are shown in Figure 7.

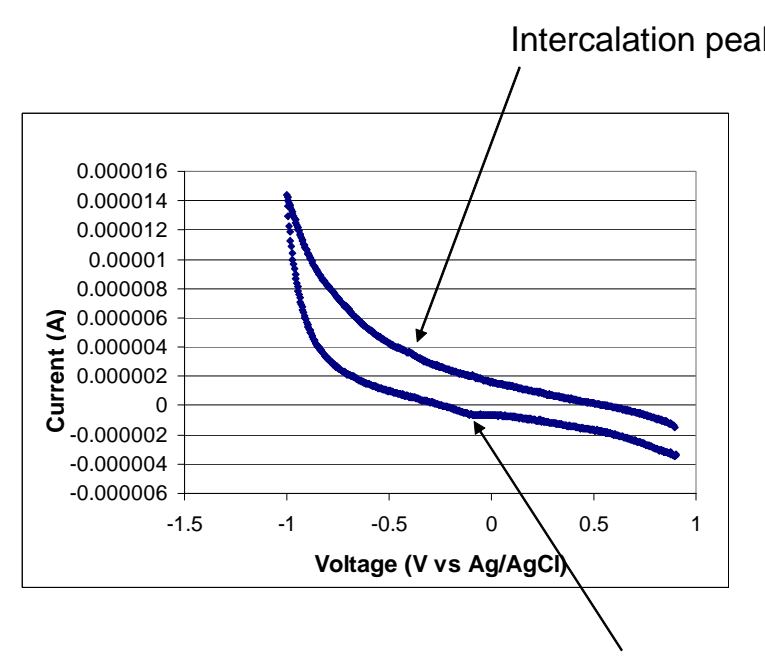

De-intercalation peak

Figure 7. $\mathrm{CV}$ of $\gamma-\mathrm{MnO}_{2}$ nanowires on HOPG electrode.

Peaks are barely discernable due to limited mass of nanowires on the electrode. Blank measurements on bare

HOPG showed no evidence of peaks. 
The CV peaks are small due to the limited mass of the nanowires. Blank measurements showed no peaks. Peak position, although shifted somewhat relative to platinum, is still in the correct voltage range.

The next step, as originally proposed, was to remove the intercalated nanowires and analyze their crystal configuration. This was not accomplished, as addressed in the following paragraphs. We had intended, following crystal characterization, to form the spinel form with high-temperature treatment.

\section{Removal of Nanowires from HOPG Surface}

The quantitative removal of nanowires from the surface of the HOPG has been an unanticipated challenge for this project. Dr. Penner's group has successfully removed nanowires after reduction to the elemental metal, but had not perfected removal of the metal oxide nanowires, which are much more brittle and apparently more adherent to the HOPG.

We investigated several techniques, but none showed quantitative transfer (i.e., transferred with high efficiency) of large quantities of nanowires from the HOPG to free-standing forms. This is a key requirement for conversion of nanowires to the spinel $\mathrm{LiMn}_{2} \mathrm{O}_{4}$ form or for use of the lithiated nanowires as cathode material.

The techniques investigated included removal with ice, scotch tape, cast PVDF of different thicknesses, melted thermoplastics of different types (polyethylene and Surlyn), wiping with different media (cotton swabs, Teflon-coated fiberglass, polyester 'mosquito' netting, Kimwipes), scraping with an Xacto knife, and ultrasonication in various solvents and configurations. All techniques were capable of removing the nanowires to various degrees. Results are summarized in Table 1.

Table 1. List of Removal Techniques Investigated and Results

\begin{tabular}{|ll|}
\hline Technique & Results \\
\cline { 2 - 2 } Thin film cast PVDF & Cleaved graphite plane \\
Thick film cast PVDF & Released clean, but limited nanowire transfer \\
Ice & Released clean, but limited nanowire transfer \\
Surlyn film melt & Cleaved graphite plane \\
Polyethylene film melt & Cleaved graphite plane \\
Cotton swap & Removed nanowires cleanly, nanowires difficult to remove from cotton \\
Xacto knife & Large amount of graphite removed \\
Teflon coated fiberglass & Significant graphite removed \\
Polyester netting & Fairly clean removal, couldn't remove nanowires from polyester \\
Kim wipe & Best clean nanowire removal, couldn't remove nanowires from wipe \\
Ultrasonic & Too aggressive, significant graphite flaking \\
\hline
\end{tabular}

The best removal of nanowires (with minimal graphite) was achieved by the wiping techniques (polyester and Kim-wipes). One encouraging idea is to use a wipe technique with a wipe that could be subsequently dissolved in a solvent, thus leaving nanowires. No attempts to try this technique were done. 
Although that fact the nanowires can be physically removed is encouraging, the techniques were not refined enough to suggest we had achieved quantitative removal. This remains a key technical challenge. "Harvesting" is an issue for many nanomaterials fabricators.

\section{Conductivity Issues}

In contrast to previous observations with nanowire growth of $\mathrm{MoO}_{2}$ and noble metals (e.g., $\mathrm{Cu}$, $\mathrm{Ag}, \mathrm{Au}$ and $\mathrm{Ni}$ ), the $\mathrm{MnO}_{2}$ nanowires did not display increases in nanowire diameter proportional to time $\mathrm{e}^{1 / 2}$. Instead, the growth followed $\mathrm{t}^{1 / 2}$ for the first $150 \mathrm{~s}$. After this point a negative deviation was observed, as shown in Figure 8.

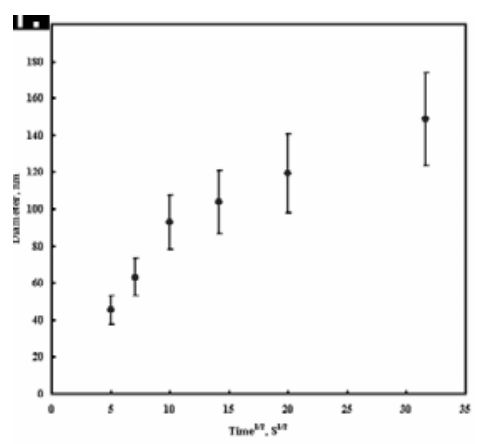

Figure 8. Negative deviation from $\mathbf{t}^{1 / 2}$ proportionality between growth of nanowires diameter and time. We believe this is due to poor conductivity of the $\gamma-\mathrm{MnO}_{2}$, which inhibits electrodeposition.

We believe that this growth inhibition of larger diameter nanowires is due to the poor conductivity of the $\gamma-\mathrm{MnO}_{2}$. This factor can have important implications for the use of these materials for high-power batteries as well.

Similar conductivity characteristics for the spinel $\mathrm{LiMn}_{2} \mathrm{O}_{4}$ have been indicated in literature accounts ${ }^{(2)}$. The spinel has been found to have semiconducting properties for the useful operating voltage ranging from $\sim 2.4 \times 10^{-5} \mathrm{~S} / \mathrm{cm}$ at $3.5 \mathrm{~V}$ to $\sim 1.3 \times 10^{-5} \mathrm{~S} / \mathrm{cm}$ at $4.2 \mathrm{~V}$ (vs. lithium), with no transition to metallic conductivity as observed for $\mathrm{LiCo}_{2} \mathrm{O}_{4}$ and some of the other lithium spinels like $\mathrm{LiTi}_{2} \mathrm{O}_{4}$ and $\mathrm{LiV}_{2} \mathrm{O}_{4}$.

These facts suggest that the use of conductive additives (carbon black) will still be required for spinel nanowires.

\section{Scaleup of Nanowire Synthesis}

In general, no fundamental technical barriers have been found that would prevent the scaleup of the electrodeposition of nanowire cathode materials. This can be accomplished by using larger HOPG electrodes. Rough calculations show that about $1 \mu \mathrm{g}$ of $100 \mathrm{~nm}$-diameter nanowires $/ \mathrm{cm}^{2}$ can be synthesized in one two-minute deposition. With an electrode area of $1,000 \mathrm{~cm}^{2}(10 \mathrm{~cm} \mathrm{x}$ $10 \mathrm{~cm}), 1 \mathrm{mg}$ could be made in one deposition. Commercial plating baths can have electrodes of $1 \mathrm{~m}^{2}$ or larger, which would then produce $0.1 \mathrm{~g}$ per deposition. Considering one $1 \mathrm{~m}^{2}$ plate and a 10 minute turnaround, up to $14.4 \mathrm{~g}$ could be produced in a day. Increasing production from this point would be a matter of increasing the number of $1 \mathrm{~m}^{2}$ plates. 
A key factor for this approach remains the removal of the nanowires. It is important to identify rapid removal techniques with quantitative removal to facilitate scaleup. No attempts to evaluate the economic ramifications of the scale-up were made in Phase I, however, prospects for an inexpensive cathode material based on these techniques are poor.

\section{Impact of Project Changes and Challenges on Phase I Objectives}

Our major Phase I end objective was to demonstrate the feasibility of forming nanowire cathode materials for high-power EV/HEV lithium-ion batteries. In large part, the project has been successful, in that we accomplished the formation of $\gamma-\mathrm{MnO}_{2}$ nanowires. However, not all the tasks and interim objectives originally proposed were achieved, due to two primary reasons:

1. We purposefully modified the objectives of the project in an attempt to produce spinel $\mathrm{LiMn}_{2} \mathrm{O}_{4}$ nanowires, rather than the lower-voltage orthorhombic $\mathrm{Li}_{\mathrm{x}} \mathrm{MnO}_{2}$ originally proposed. This was in direct response to suggestions made by one of the Phase I reviewers and we felt the potential benefits associated with the spinel form were worth redirecting resources from the originally planned tasks. As expected, the more aggressive effort reduced the time and resources available for accomplishing all of the original objectives. We believe we made good headway toward achieving the spinel synthesis and see no substantive reasons why it cannot be accomplished as a future research challenge.

2. We underestimated the challenges associated with the various phases of nanowire growth, their removal from the HOPG electrode surfaces and their subsequent manipulation. The transfer of the nanowire synthesis accomplished at UCI to Boundless was also poorly planned. Boundless' experience and Phase I research have now familiarized us with many of the challenging issues that are very common in the field of nanotechnology. This valuable learning experience should make us better suited to plan for and address such issues in a knowledgeable manner, in future research.

\section{Conclusions}

Given our experiences in Phase I, a realistic goal for future research will be to demonstrate the synthesis of spinel $\mathrm{LiMn}_{2} \mathrm{O}_{4}$ nanowires and demonstrate their performance (including cycle life) in lithium-ion batteries. The challenge of scaleup feasibility must also be addressed. If the promises of this technology (including economic factors) are proven, it is realistic to expect strong commercial interest and subsequent third party funding for commercialization.

\section{References}

1. E. Levi, E. Zinigrad, H. Teller, M.D. Levi, D. Aurbach, E. Mengeritsky, E. Elster, P. Dan, E. Granot, H. Yamin, J. Electrochem. Soc. 144, 4133 (1997).

2. S. Yamamura, H. Koshika, M. Nishizawa, T. Matsue, I. Uchida, J. Solid State Electrochem. 2, 211-215 (1998). 